\title{
Experimental Investigation of Local Dynamics in a Bolted Lap Joint Using Digital Image Correlation
}

Brøns, Marie; Kasper, Thomas A.; Chauda, Gaurav; Klaassen, Steven W. B.; Schwingshackl, Christoph W.; Brake, Matthew R. W.

Published in:

Journal of Vibration and Acoustics

Link to article, DOI:

10.1115/1.4047699

Publication date:

2020

Document Version

Peer reviewed version

Link back to DTU Orbit

Citation (APA):

Brøns, M., Kasper, T. A., Chauda, G., Klaassen, S. W. B., Schwingshackl, C. W., \& Brake, M. R. W. (2020). Experimental Investigation of Local Dynamics in a Bolted Lap Joint Using Digital Image Correlation. Journal of Vibration and Acoustics, 142(5), [051114]. https://doi.org/10.1115/1.4047699

\section{General rights}

Copyright and moral rights for the publications made accessible in the public portal are retained by the authors and/or other copyright owners and it is a condition of accessing publications that users recognise and abide by the legal requirements associated with these rights.

- Users may download and print one copy of any publication from the public portal for the purpose of private study or research.

- You may not further distribute the material or use it for any profit-making activity or commercial gain

- You may freely distribute the URL identifying the publication in the public portal 


\title{
Experimental investigation of local dynamics in a bolted lap joint using digital image correlation
}

\author{
Marie Brøns* \\ Thomas A. Kasper \\ Department of Mechanical \\ Engineering \\ Technical University of Denmark \\ Nils Koppels Allé 404, 2800 Kgs. Lyngby \\ Denmark \\ Institute of Dynamics and Vibration Research \\ Leibniz Universität Hannover \\ Appelstr. 11, 30167 Hannover \\ Germany \\ Email: kasper@ids.uni-hannover.de \\ Email: maribr@mek.dtu.dk \\ Gaurav Chauda \\ Department of Mechanical \\ Engineering \\ Michigan State University \\ East Lansing, Ml 48824 \\ Email: chaudaga@egr.msu.edu
Christoph W. Schwingshackl
Imperial College London
South Kensington Campus, \\ London SW7 2AZ \\ United Kingdom \\ Email: c.schwingshackl@imperial.ac.uk \\ Steven W. B. Klaassen \\ Institute of Applied Mechanics \\ Technische Universität München \\ Boltzmannstr. 15, 85748 Garching \\ Germany \\ Email: steven.klaassen@tum.de \\ Matthew R. W. Brake \\ William Marsh Rice University \\ 6100 Main St., Houston, TX 77005 \\ Email: brake@rice.edu
}

\begin{abstract}
The dynamics of structures with joints commonly show nonlinearity in their responses. This nonlinear behaviour can arise from the local dynamics of the contact interfaces. The nonlinear mechanisms at an interface are complicated to study due to the lack of observability within the contact interface itself. In this work, Digital Image Correlation (DIC) is used in combination with a high-speed camera to observe the local motion at the edge of the interface of a bolted lap joint. Results demonstrate that it is possible to use this technique to monitor the localised motion of an interface successfully. It is observed that the two beam parts of the studied lap joint separate when undergoing bending vibrations, and that there is a clear asymmetry in the response of the left and the right end of the interface. Profilometry indicates that the asymmetry in the response is due to the mesoscale topography of the contact interface, highlighting the importance of accounting for surface features in order to model the nonlinearities of a contact interface accurately.
\end{abstract}

${ }^{*}$ Address all correspondence to this author. 


\section{Introduction}

The accuracy of structural dynamic models is often limited to the least representative part, especially if that part is in the mechanical load path (such as joints). Furthermore, validation of such models is typically made against a global response, since there is scarce knowledge of the local response of a joint. While accurate dynamic models of structural components are becoming commonplace, accurate modeling of the component-to-component interactions is still challenging. One common shortcoming of predictive models of joints is the lack of understanding of the mechanisms underlying the observed nonlinear behaviors (such as resonance shifts, change in dissipation, harmonics, etc.) that exist even in simple joints.

Various nonlinear joint models can be constructed and implemented for different contact cases [1,2], but without supporting data or experimental validation, these models are generally unreliable. Some methods try to circumvent this problem by calibrating joint models with experimental data; either by measuring or optimizing joint parameters [3-5], or by including the implicit macro effects of the joints in the component models [6]. However, using measurement data is, by definition, non-predictive and thus requires expensive prototypes. Furthermore, the use of noisy measurement data results in large uncertainty bands [7]. An alternative approach is to model the joint in (relatively) high fidelity to predict the nonlinear behavior, such as in $[8,9]$ where high fidelity contact models of interfaces in aeroturbine assemblies are used to make predictions of vibration amplitudes and contact forces, or in [10] where the mesoscale features of the interface are used to make blind predictions of the dynamics of a structure with a lap joint.

In most cases, bolted joints introduce a strong damping nonlinearity and a weak stiffness nonlinearity to a structure [11-13]. Because the weak stiffness noninearity is often amenable to the assumption that modes shapes do not change and that there is little modal coupling, much research has focused on developing constitutive models to represent the hysteretic behavior of an interface [14-17].

In many structures damping is beneficial $[15,18]$ (e.g., in built-up structures, damping can prevent unwanted vibrations), and is often caused by energy dissipation arising from micro-slip internal to frictional interfaces. Therefore, it is of great interest to be able to predict and model such micro-slip accurately, which requires further insight in the local dynamics of a joint.

Measurements or observations of a joint can enhance the understanding of the physical mechanisms that cause nonlinear behaviors, such as sticking, slipping (micro- or macro-), and separation (partial or full). Once these mechanisms are understood, the physics included in nonlinear models can be improved based on the experimental observations, creating a new basis for future joint studies. This requires that the following apply:

1. The measurements can capture the different interface contact effects: sticking, slipping, and separation; the relative displacements are above the measurement noise floor (sensitivity); and different effects are distinguishable (observability).

2. The measurements are repeatable. Any measured change throughout multiple measurements of a nominally identical setup should be negligible when compared to the measured contact effects. If this is not the case, no conclusion can be made on the correlation between cause (mechanisms) and effect (behavior). 
Various techniques have been applied in order to capture these effects, the majority of which use a force excitation to impose joint displacements that are then directly or indirectly measured. One direct technique is to use accelerometers on or near the interface [3]. However, the physical nature of interfaces, and the fact that the sensors change the dynamic properties of the system, limits the practical use of this technique. Laser Doppler Vibrometers (LDVs) can circumvent these problems as they add no significant physical effects and are non-contact based measurement techniques. In [19], a method is presented that compares the overall dynamics of a bolted structure to that of an unbolted, but otherwise similar one. The LDV measurements indicate the presence of non-proportional damping and non-linear effects in the bolted structure due to microimpacts of the connected beams at the bolted joint. However, to measure the required in-plane motion, a typically expensive 3D scanning LDV (SLDV) is needed. In [20], two SLDVs were used on a flange joint undergoing bending vibration, and the local motion at the joint was monitored. It was found that the nonlinear mechanism, here especially the damping, was different for different flange geometries. Furthermore, an LDV measures only a single point at a time; to obtain a high-spatial resolution, the interface has to be excited for a prolonged time, which may result in wear [21].

Recently, digital image correlation (DIC), in conjunction with a high-speed camera, has been used to observe physical effects in structures [22]. The same technique has also been used to investigate the shock and vibration of the electronic assemblies, where accurate, full-field measurements have been obtained as a method to monitor in-situ health of electronic assemblies [23]. For single-lap, bonded joints, local strains were successfully measured using DIC to gain insights into crack formation and growth $[24,25]$. With this technique, the in-plane displacements of various pixel-groups in the images can be tracked in a single measurement, ensuring a relatively high-spatial resolution. However, since this technique measures displacements rather than accelerations, it is limited to a low-frequency bandwidth ${ }^{1}$.

In this work, high-speed DIC is used to observe the contact motion effects of a bolted lap joint in an altered version of an academic testing structure [26]. Together with the subsequent work in [27], this research presents a novel combination of high-speed DIC and detection of sources of nonlinearity in jointed structures. The technique is applied successfully to a bolted lap joint, revealing its potential in being a new way to experimentally investigate sources of nonlinearity in many types of joints and structural connections as it allows for nonintrusive testing. The Brake-Reuß beam (BRB) benchmark $[13,26]$ is specifically designed to show nonlinear effects at relatively low natural frequencies. A longer version is used for this research, creating larger deflections on the interface at even lower natural frequencies, making it an appropriate case for DIC. The BRB has been used in the past to observe nonlinear mechanisms at the contact interface. In [26,28,29], different shapes and geometry of the beam have been used to analyze the effect of interface geometry on natural frequencies and damping, and in [30] electronic pressure films and sensors have been used to locate the high- and low-pressure regions at the interface to monitor the dynamic pressure levels during a vibration cycle. Electronic pressure film sensors give great insights into joint behavior, but are an intrusive technique and may change the behavior of the joint. Notably, one of the main observations

\footnotetext{
${ }^{1} \mathrm{~A}$ force-controlled shaker's input is proportional to the acceleration and not the displacement. The ratio between displacement and acceleration amplitudes diminish by two orders per decade and thus, at higher frequencies, the displacement amplitudes are immeasurably low, for most measurement techniques
} 
of [30] was that in measuring the contact pressure during dynamic excitation, there was evidence of significant separation in the interface near the edges of the interface. The finding led to the hypothesis that there are significant local kinematics (i.e., clapping and separation) within the interfaces of the bolted joints. This research seeks to test this hypothesis through direct optical measurements of the local joint motion during bending vibrations in a lap joint.

The objective of this work is to obtain experimental measurements of the nonlinear mechanisms of an excited structure containing a jointed surface. To do this, DIC, together with high-speed videography, is used to capture the vibrations along the interface edge of the joint. Section 2 explains the experimental setup in detail, along with the essential practical considerations. The various methodology choices that are made for the high-speed camera and DIC procedure are elaborated in Section 3. The results obtained with these procedures in the proposed test-cases are highlighted in Section 4. The observations are critically discussed in Section 5, and conclusions are given in Section 6.

\section{Experimental Setup}

\subsection{Test specimen \& setup}

The Brake-Reuß Beam (BRB) system is a benchmark structure for studying joint-based nonlinear behavior [13,26]. An altered version of the nominal BRB is used as a test-case; both sides of the beam are $180 \mathrm{~mm}$ longer than the nominal beam (described in [26]), but the interface geometry is the same. The altered beam is displayed graphically in Figure 1. This alteration ensures larger interface displacements at lower natural frequencies as compared to the nominal BRB, which is a useful characteristic for the DIC study - since displacements are measured as opposed to accelerations. The BRB consists of two stainless steel 304 alloy beams, each with a square cross section of $25.4 \mathrm{~mm}$ ( 1 inch) side length. The beams have been manufactured by wire electrical discharge machining. The surfaces have been ground to at least an $8 \mu \mathrm{m}$ finish. In inspecting these beams before testing, the surface finish was measured to have mean roughness of approximately $1 \mu \mathrm{m}$. These beams are connected via a bolted lap joint, using three grade 5 5/16"-24 bolts as seen in Figure 1. In the follow on work [27], experiments are performed on other non-flat interfaces (concave and convex shapes).

In order to satisfy the repeatability condition in the experiment, the bolts are tightened using a procedure explained in [26]: The beams are mated, aligned against two flat surfaces, and clamped such that they do not move while tightening. To ensure that the axial alignment is consistent, a shim with a fixed thickness $(0.2 \mathrm{~mm})$ is used while tightening, as shown in Figure 1. First, the center bolt is tightened to $70 \%$ of the final bolt torque value, then each of the outside bolts are tightened to the same level. When all three bolts have been tightened to this intermediate level, they are subsequently tightened to the final torque, in the same order. Three different bolt torque levels are tested; 5, 10, and $20 \mathrm{Nm}$, which corresponds to approximately 15,30 , and $60 \%$ of the bolts' yield strength. However, the stress percentages are rough estimates, as the bolt torque relation to preload is controlled by a nut factor, which is very sensitive to the material coatings and lubrication [31]. Nonetheless, it indicates how close the applied tightening is to industrial tightening levels. No lubrication was used in the experiments, thus the nut factor is specified as 0.2 . US grade 5 bolts are used, with the yield strength of $634 \mathrm{MPa}$ and a 


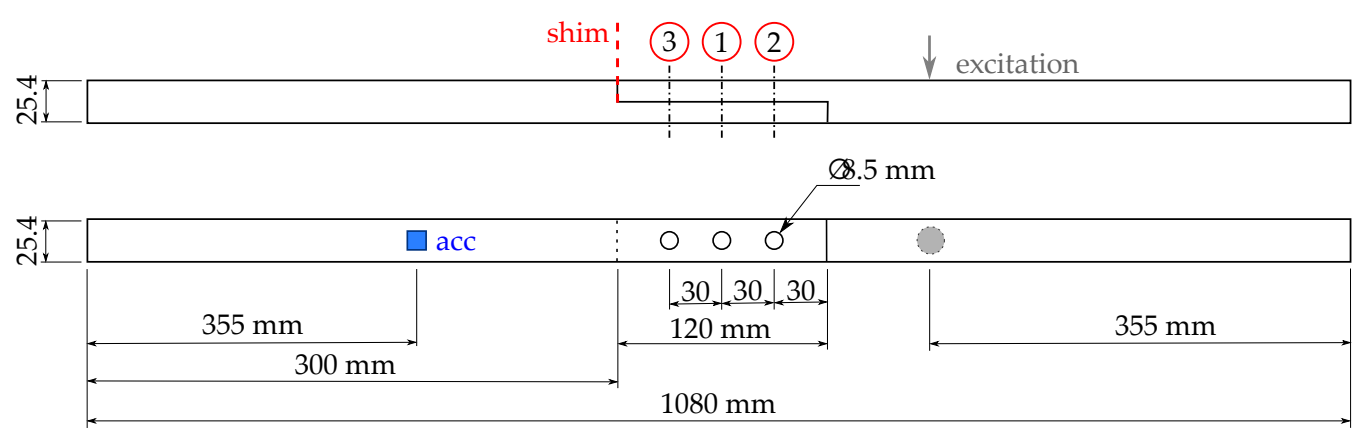

1-axis accelerometer shaker position ---shim and tightening order

Fig. 1. A sketch of the extended Brake-Reuß beam used for the measurements. The bolt tightening order is shown. A gap of $0.2 \mathrm{~mm}$ is ensured on the left side of the interface by means of the shim.

stressed area of $31.8 \mathrm{~mm}^{2}$ [31]. For industrial applications, it is common to tighten bolts to around 70-80\% of their yield strength [31]; this has purposely been avoided in these experiments to prevent too much wear on the beam surfaces, as the setup was re-tightened numerous times.

As shown in Figure 2a, a small shaker $(15 \mathrm{~N})$ is used to excite the structure at resonance for the different modes of the beam. The shaker is placed away from the joint, to avoid potential disturbance from the shaker at the joint, and also away from first bending mode nodal points. The shaker is force-controlled at different levels of force to study the effect of excitation level on the interface motion. To assess the influence of the shaker location, a series of control tests were conducted in which, the shaker was placed on the other side of the beam, and below instead of above the beam (in all tests, the shaker was located at the same distance from the interface to not change the level of modal excitation). Neither of these configurations significantly affected the results, which demonstrated that the shaker position is not influencing the observed bending vibrations of the beam. A uni-axial accelerometer is positioned at the other side of the beam to capture the response of the system (Fig. 1,2). As shown in Figure 2, the entire setup (BRB and shaker) is suspended with fishing lines to mimic free-free boundary conditions.

\subsection{High-speed camera}

A PHOTRON FASTCAM SA2 high-speed camera is used to capture the response of the first in plane bending mode (near $80 \mathrm{~Hz}$, the $y$ direction bending mode cf. top scheme in Figure $2 \mathrm{~b}$ ) and the first out-of-plane bending mode (near $104 \mathrm{~Hz}$, the z-direction bending mode, cf. bottom scheme in Figure 2b) of the structure. The camera can produce 1000 frames per second (fps) with a resolution of $2048 \times 2048$ pixels, with higher fps resulting in lower resolution images.

To inspect the interface in detail, the camera acquired images at $2000 \mathrm{fps}$ at a resolution of $2048 \times 1080$ pixels. The main criterion for useful analysis is a decent time resolution and image size to enable the precise analysis of the first bending mode. To further increase the spatial resolution a NIKON AF-S MICRO-NIKKOR $105 \mathrm{~mm}$ lens is used to zoom-in at specific parts of the interface (cf. Figure 3 with zoom-in at areas $\mathrm{C} 1, \mathrm{C} 2$ and $\mathrm{C} 3$ ). 


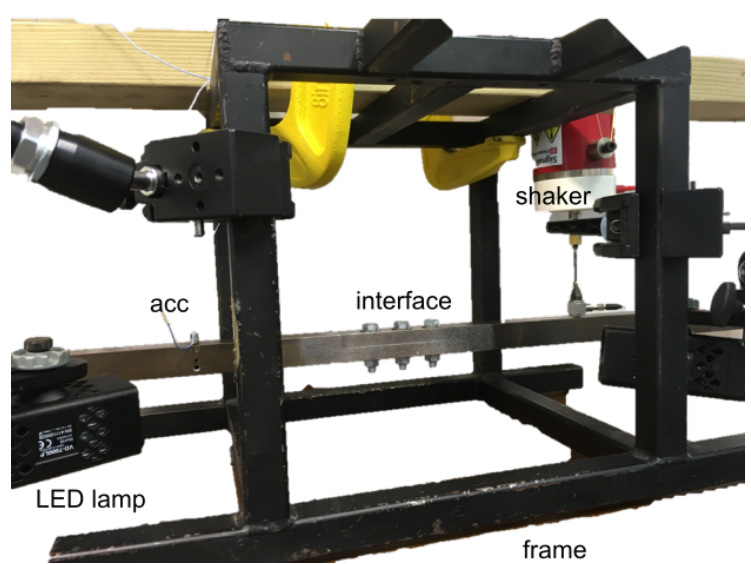

(a)
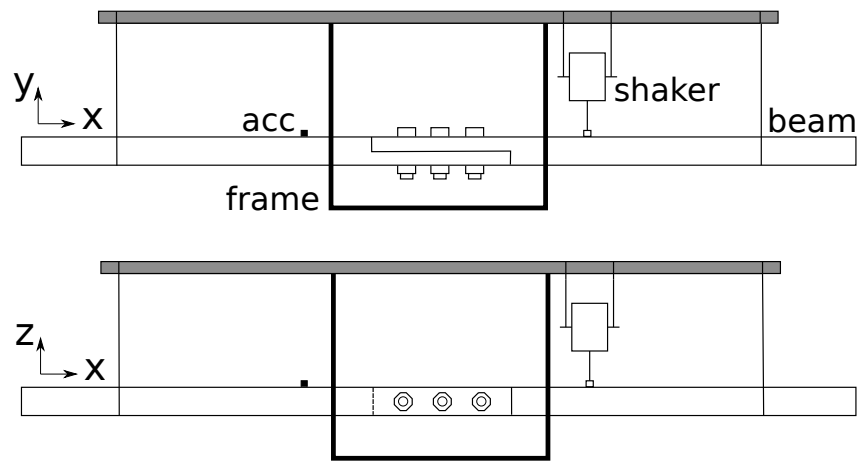

(b)

Fig. 2. (a) Real experimental setup. (b) scheme of the two configurations, in plane bending ( $y$ direction) and out-of-plane bending ( $z$ direction), with the beam and shaker suspended with fishing line.

Water based white spray paint is sprayed on the beam surface near the interface surface to create a speckle-pattern with a desired speckle-size ranging from 3 to 5 pixels [32]. Subsequently, the interface surfaces were thoroughly cleaned to remove any ingress of paint. As a consequence of the high rate of image capturing, the amount of light captured by the camera per frame is reduced (the exposure time was $145 \mu$ at $2000 \mathrm{fps}$ ). Therefore, the camera's field of view is illuminated using two high intensity LED lights as shown in Figure 2a.

\subsection{Experimental Procedure}

The BRB is excited with shaker excitation at the natural frequency of the investigated mode. To identify the desired excitation frequency, a hammer-impact test is first performed. Since the natural frequencies can shift slightly for each assembly, a frequency sweep with a bandwidth of $5 \mathrm{~Hz}$ around the expected natural frequency is performed in order to find the assembly-specific natural frequency. Afterwards, the BRB is excited at this frequency for a $10 \mathrm{~s}$ period during which the camera is manually triggered. The camera captures 2589 images during the measuring time of $1.3 \mathrm{~s}$, resulting in 103 vibration cycles of the first bending mode. Steady state conditions allow for a subset of 100 frames. As the primary mode of investigation is the first bending mode near $80 \mathrm{~Hz}, 100$ frames spans four cycles of vibration. For those tests, the shaker is vertically suspended (cf. top scheme in Figure 2b). In the follow on work [27], experiments are performed both the first and second in-plane bending modes for a similar system with a lap joint. For another comparison, the out-of-plane bending mode (near $104 \mathrm{~Hz}$ ) is tested in this work, and for these tests the setup is rotated 90 degrees so that the shaker excites in the $z$ direction (cf. bottom scheme in Figure 2b). The experiments test both the influence of bolt-tightening and excitation level on contact behavior of the structure. The resolution is increased significantly by zooming in on the individual areas $\mathrm{C} 1, \mathrm{C} 2$ and C3 (see Figure 3) instead of recording the entire joint. 


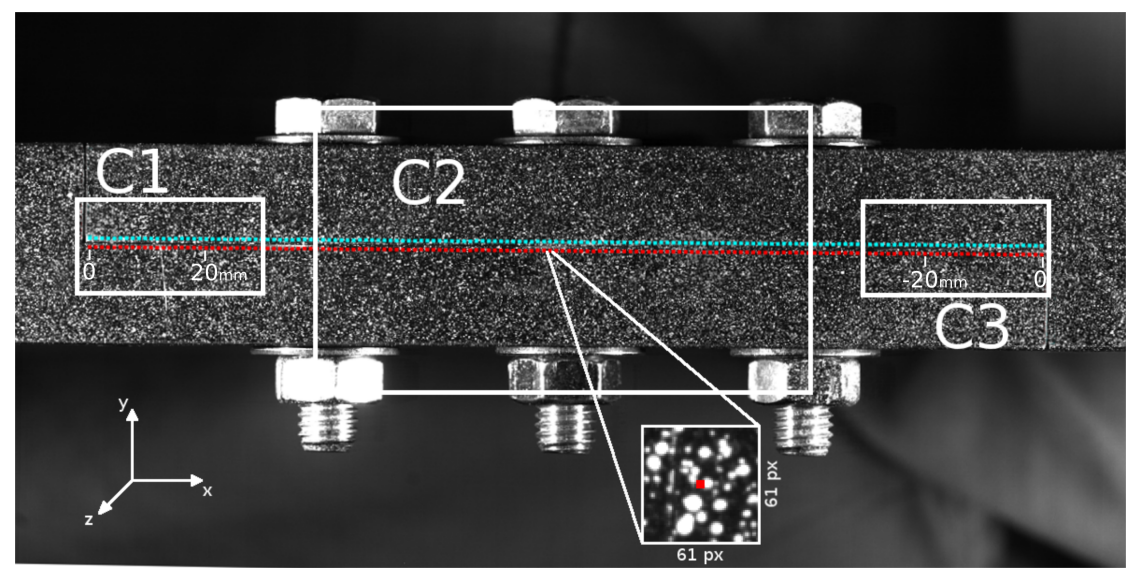

Fig. 3. A sample frame from the high-speed camera. The three different areas of interest $(\mathrm{C} 1, \mathrm{C} 2, \mathrm{C} 3)$ are shown. The relative displacements of the interfaces (defined by the subsets in the red and blue line along the contact-line) will be measured. A zoom-in of a typical $61 \times 61$ pixel subset and the principle of subsets, step size and overlap is also shown.

\section{Methods}

\subsection{Digital Image Correlation}

DICE (Digital Image Correlation engine) [33] is a free to use DIC software package from Sandia National Laboratories. Here, it is used in this project to extract the displacements of several points along the beam's interfaces from the high-speed camera images. In order to find the displacements of the pixel subsets, DICE requires a reference image to compare every frame to. In this case, the first image of the extracted set is used as the reference; since the extraction is arbitrary, the reference image is generally not of an equilibrium position. Therefore, the equilibrium position needs to be found in the post-processing.

Instead of analyzing the whole image - which would take a considerable amount of time - a line of pixel subsets is created on both sides of the interface (cf. the red and blue lines in Figure 3). There are two parameters to be chosen for the DIC measurements: the subset size and the step size of that subset. Both concepts are illustrated in Figure $4 \mathrm{~b}$. A brief study is conducted on the effect of subset-size to the standard deviation of the displacement measurement, shown in Figure 4a. An approximately reciprocal relationship between subset size and DIC processing error (as quantified by the standard deviation) is evident. It must be noted that even though - when looking at DIC error - a larger subset size is always better, it is not always preferable: a larger subset size diminishes spatial resolution (amount of subsets along the line) and masks the flexible motion of the beams. Based on this study, a subset size of $61 \times 61$ pixels is selected, with a step size of 41 pixels; this ensures $66 \%$ subset overlap, which agrees with the recommendations in [34]. These settings ensures both a minimal displacement noise and a sufficient spatial resolution (43 subsets along the $\mathrm{C} 1$ interface edge). An overlap prevents the search algorithms from diverging, provides a high spatial resolution, and allows a dependent correlation with other subsets to speed up the DIC processing analysis. A gradient-based optimization technique is used as the search algorithm with initialization based on its previous field values (i.e. displacement) at each step. It is the most common in the literature and is recommended for cases without large rotations, initial guess instabilities, or costly iterations due to large deformations [35]. This DIC procedure 


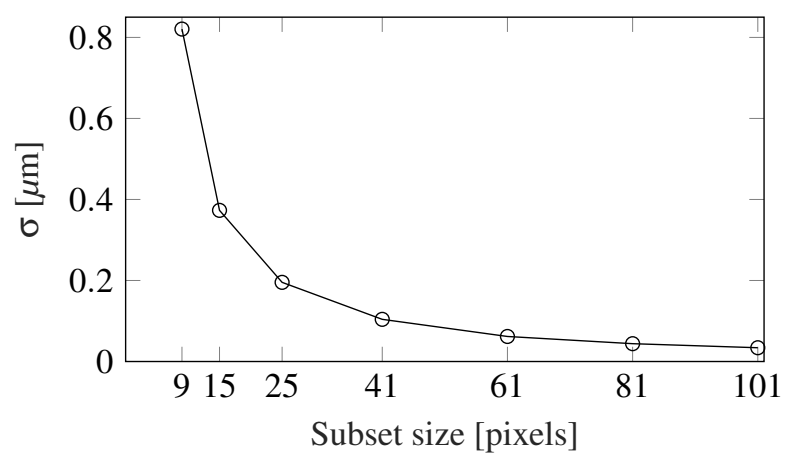

(a)

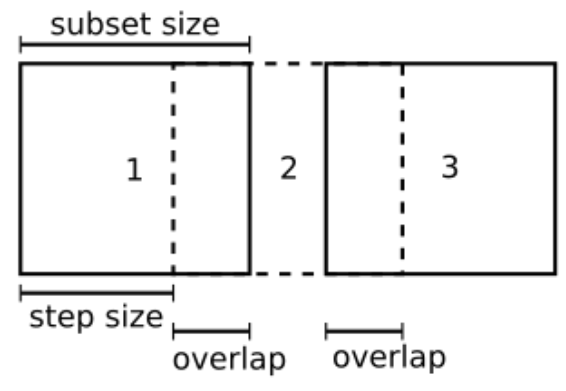

(b)

Fig. 4. (a) The standard deviation of the relative displacement measurement for different subset sizes. (b) Illustration of relation between subset size, step size and overlap.

results in a quick and accurate analysis, allowing for a set of 100 images to be analyzed in a matter of seconds. For every single image, it obtains the displacements in the $x-y$ plane of each pixel subset (dots in Fig. 3) related to a reference image. The parameters for the DIC setup are summarized in Table 1.

Table 1. DIC-Setup

\begin{aligned} & \hline Camera Type-1 PнOTRON FASTCAM SA2 \\ & Image Size $2,048 \times 1,080$ pixels \\ & Framerate 2,000 frames/s \\ & Exposure $145 \mu \mathrm{s} \\ &$ Lens NIKON AF-S MICRO-NIKKOR $105 \mathrm{~mm} \\ &$ DIC Software DICE \\ & Avg. Speckle Size $5 \times 5$ pixels \\ & Subset Size $61 \times 61$ pixels \\ & Step Size 41 pixels \\ & Correlation Algorithm gradient-based [36] \\ & Interpolation Keys fourth-order [37] \\ & \hline\end{aligned}

\subsection{Post processing}

The displacement data obtained in DICE is post processed in MATLAB to investigate the local dynamics of the interface. The main captured motion is the the global bending motion. It has four cycles within the 100 frames and there is no rigid body motion. The joint is located near an anti node of the first mode, and the amplitude is in the order of $60 \mu \mathrm{m}-250 \mu \mathrm{m}$ (depending on the level of the forcing). 
To investigate the local behavior at the interface, a relative displacement:

$$
\Delta \mathbf{u}_{i}(t)=\mathbf{u}_{i}^{\mathrm{L}}(t)-\mathbf{u}_{i}^{\mathrm{U}}(t)
$$

is computed for each subset (denoted as $i$, see Figure 3), where $\mathbf{u}^{\mathrm{L}}(t)$ and $\mathbf{u}^{\mathrm{U}}(t)$ are the displacement on the lower and upper beam at time $t$ respectively, which allows the global bending mode of the beam to be removed in order to focus on the relative motion between the two interfaces. Eq. (1) defines the relative displacements in any chosen direction. This work compares displacements in the direction of beam bending (shaker excitation direction), which corresponds to the $y$ direction for the first bending mode and the $z$ direction for the out-of-plane bending mode, cf. Figure $2 \mathrm{~b}$.

Since the reference image used in DIC is the first frame of the measurement time, it is not necessarily an equilibrium position. The relative displacement difference will always be equal to zero at $t=0$, since both $\mathbf{u}^{\mathrm{L}}$ and $\mathbf{u}^{\mathrm{U}}$ are zero at $t=0$ (the displacement between the first picture and itself is obviously zero); therefore, a reference frame shift is required.

The reference frame is fixed by calculating the mean (i.e. the DC component) of the displacement vectors $\mathbf{u}^{\mathrm{L}}$ and $\mathbf{u}^{\mathrm{U}}$ over time. The displacement vector at the frame in time closest to the mean is used as the displacement reference, and is thus subtracted from all other frames, effectively centering the global motion around zero. The relative difference can be found by using the corrected $\mathbf{u}^{\mathrm{L}}$ and $\mathbf{u}^{\mathrm{U}}$ in Eq. (1), which is then plotted in what follows.

\section{Results}

In some cases, it may be assumed that two beams connected with a lap-joint vibrate as one solid beam, thus the relative displacements between the two beam parts would be zero. The first bending mode in the $y$ direction would then, under free-free conditions, look as illustrated in Figure 5a. In that case, there is no relative displacement at the interface. The upper and lower beam are completely clamped together. However, this assumption does not apply in this case. Figure $5 \mathrm{~b}$ shows frequency response functions measured by an accelerometer for three levels of bolt torque, measured around the first bending mode, for a low-level impulse hammer impact. As the torque is increased the first natural frequency increases (approximately $2 \mathrm{~Hz}$ from $5 \mathrm{Nm}$ to $20 \mathrm{Nm}$ ). Prior experimental work on the same system [26], also shows that the natural frequency increases with increasing bolt torque and that it decreases with excitation amplitude. For a surface finish of $0.02 \mu \mathrm{m}$, and with bolt torques of $5 \mathrm{Nm}$ or higher, the natural frequency converges to a linear natural frequency with similar stiffness nonlinearities (i.e., for bolt torques higher than $5 \mathrm{Nm}$ the natural frequency changes approximately to the same extent with response amplitude). Hammer tests must be analyzed with care due to their broad band excitation; however, in [38,39], the efficacy of hammer testing versus shaker ringdown testing (where the shaker is decoupled from the system after driving it to resonance) has shown that hammer testing yields similar results as single-mode shaker excitation and ringdown testing.

Figure 6 shows an example of the relative difference in $y$ displacement between the upper and lower beam, $\Delta y$, for all 


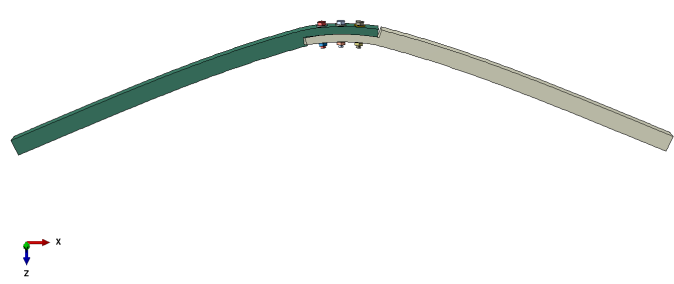

(a)

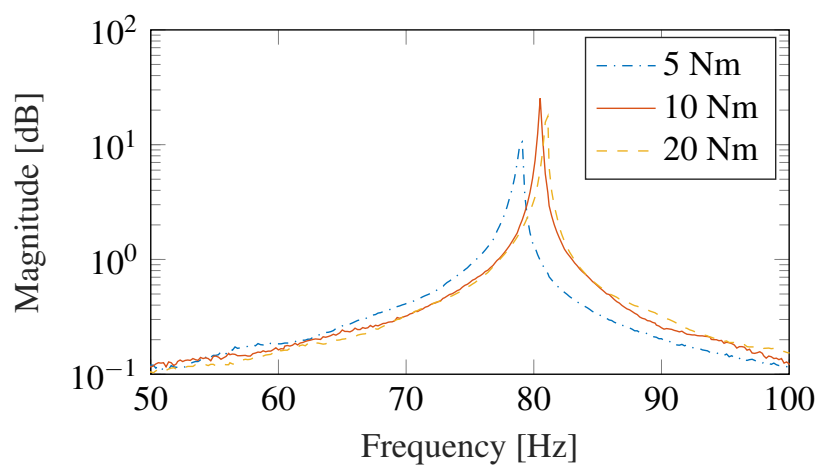

(b)

Fig. 5. a) Illustration of the first bending mode of the beam. b) Frequency response functions measured by low level hammer impact around the first bending natural frequency at different bolt torque levels

three interfaces, $\mathrm{C} 1, \mathrm{C} 2$ and $\mathrm{C} 3$. The ranges of $\Delta y$ are different for each of the sub-figures to make it more clear to see how each interface moves. For each part of Figure 6a, the beam is driven at the first bending mode by a $4 \mathrm{~N}$ shaker forcing, and the bolts are tightened to $20 \mathrm{Nm}$. In Figure 6, the displacement of the horizontal interface at $\mathrm{C} 1$ shows that a relative displacement exists and that it periodic at the same frequency as the excitation frequency. The symmetry indicates that the two interfaces do not touch at any time during the vibration cycle, suggesting that an initial gap must exist, that never closes with the applied excitation amplitude. Separation in micrometer range can be seen with increasing distance from the bolt, with a maximum relative displacement at the edge of the interface. This is in contrast to the behavior of the other side of the interface: a closing contact can be observed at $\mathrm{C} 3$ in Figure $6 \mathrm{~b}$, where the absolute minimum is nearly zero, and the magnitude of separation is about five times smaller compared to C1 (cf. Figure 6a). The recorded data is repeatable across multiple experiments.

Figure $6 \mathrm{c}$ shows the relative displacement in the $y$ direction at $\mathrm{C} 2$, which represents the region containing the bolts. Cyclic motion is not observed for $\mathrm{C} 2$ when subtracting the displacements of the upper and lower beam parts. No systematic separation can be seen, and the relative displacements are beneath the noise floor. To capture the whole C2 interface (cf. Figure 3) the resolution is lower than at $\mathrm{C} 1$ and $\mathrm{C} 3$, which makes the noise floor higher in this case. Another indicator that the measured relative displacement is noise is that the few spikes of $2 \mu \mathrm{m}$ are constant in time. Based on Figure $6 \mathrm{c}$, it is assumed that at $\mathrm{C} 2$ the beam is effectively rigidly connected, with no relative normal motion. No further analysis of the $\mathrm{C} 2$ interface is performed.

By calculating the relative displacement in the longitudinal direction ( $x$ direction), it is also possible to determine a potential sliding of the interfaces when the beam bends in the $y$ direction. There is no measurable sliding in the region containing the bolts (C2), but for $\mathrm{C} 1$ and $\mathrm{C} 3$, there is sliding. The maximum sliding is in the same order of magnitude at $\mathrm{C} 1$ and $\mathrm{C} 3$, approximately $\pm 2 \mu \mathrm{m}$. The sliding at $\mathrm{C} 1$ and $\mathrm{C} 3$ exhibit the same motion. There is no asymmetry, such as seen for the separation in the $y$ direction (cf. Figure 6). Bolt torque does not influence the magnitude of sliding, but stronger excitation gives slightly more sliding of the interfaces. These initial results show that high-speed videography combined 


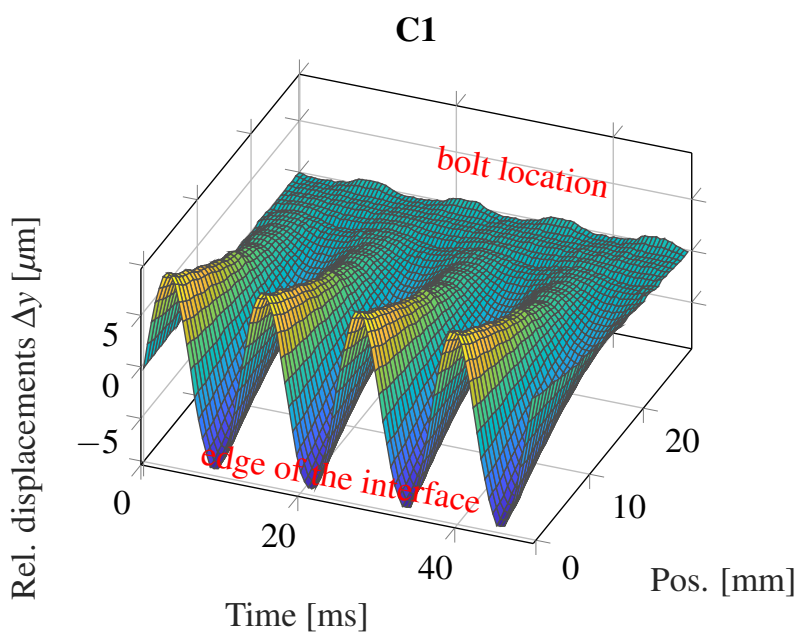

(a)
C3

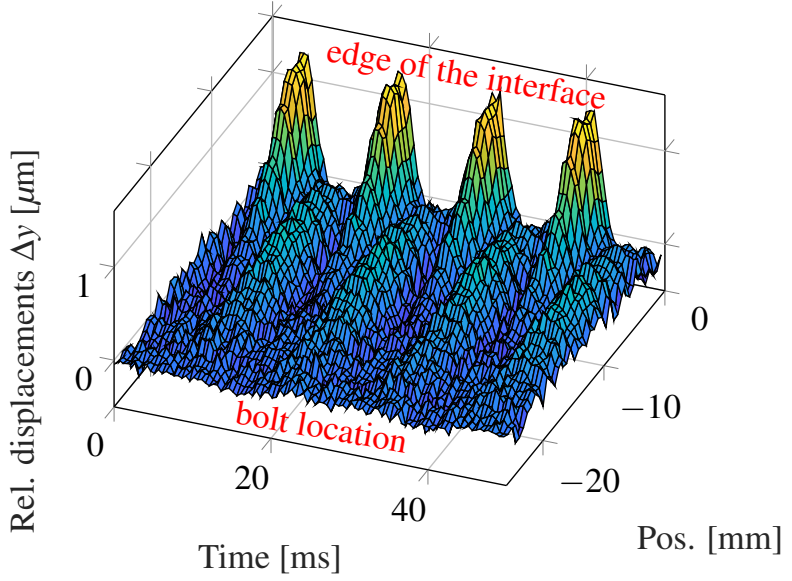

(b)

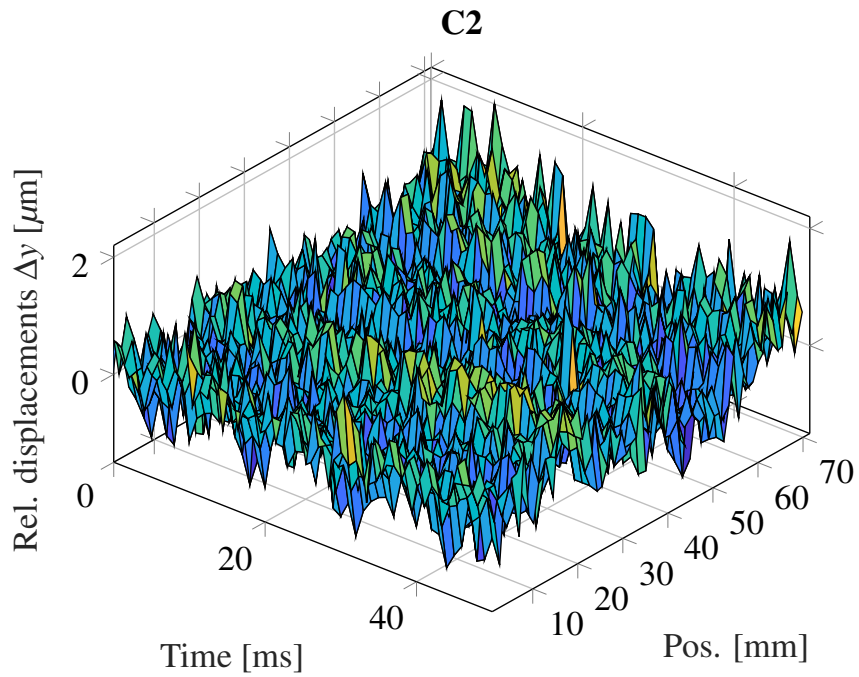

(c)

Fig. 6. Relative $y$ displacement field of the horizontal interfaces. at a) C1, b) C3, and c) C2.

with DIC is a very good technique for detecting local motion as small as a single micron. In the following, the technique is used to explore how the relative displacements at the interface depend on forcing and bolt torque levels.

\subsection{Influence of forcing magnitude}

When the first bending mode is at its maximum response amplitude (globally), the local relative motion also exhibits maximum relative displacement. The local coordinate system is defined such that the edge of the interface is at position zero, and the first bolt is located at approximately $30 \mathrm{~mm}$ (cf Figure 3). In Figure 6a, four cycles of vibrations are measured. From the envelopes of this vibration, the maximum and minimum relative displacements are calculated. The four maximums and the four minimums that correspond to the peak amplitude of the response envelope are then averaged to obtain a single maximum and minimum curve as depicted in Figure 7a, independent of time. This implicitly assumes that the relative motion 


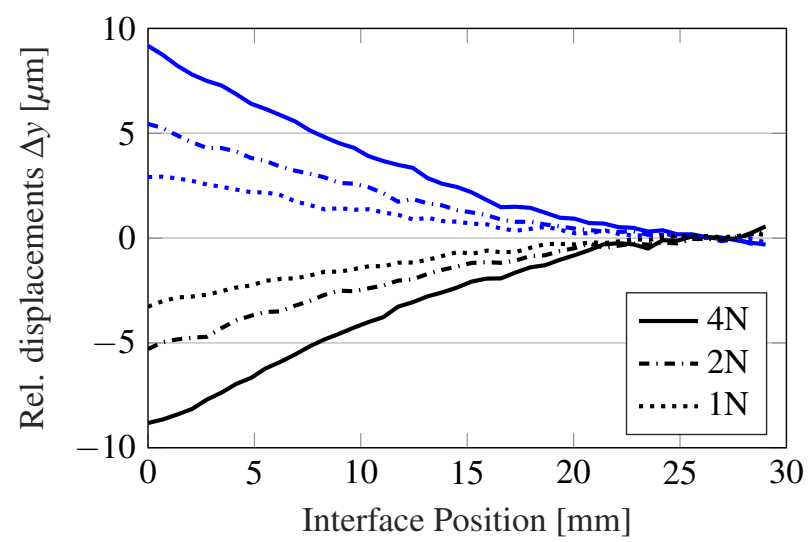

(a)

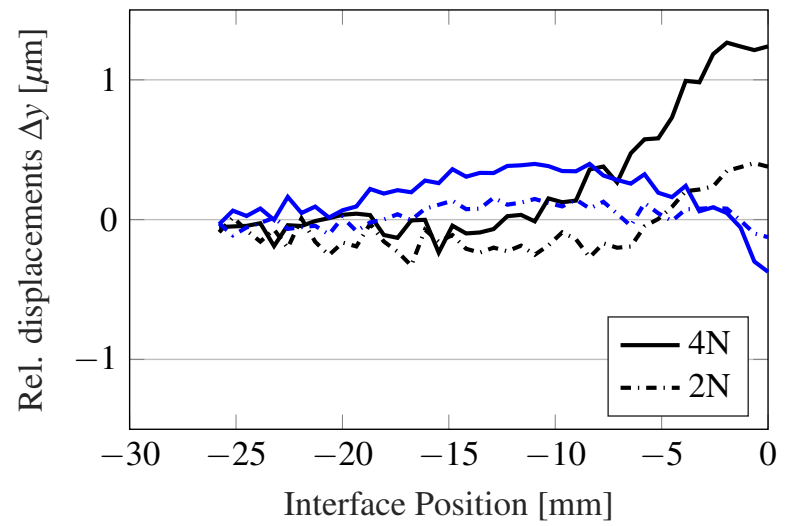

(b)

Fig. 7. Maximum relative displacement for different forcing magnitudes, with $20 \mathrm{Nm}$ bolt torque. Blue lines: Upper bending mode position (cf. Figure 5a). Black lines: Lower bending mode position (half a vibration cycle later). a) Interface C1. b) Interface C3.

is harmonic and that all cycles are steady state. This assumption was validated by comparing the average values calculated for one set of 100 frames against those from a different set of 100 frames (out of the total sampled 2300 frames per data set) for several of the reported measurements. The differences found between the average values from the different sets of 100 frames was beneath the noise floor of the measurements. Figure 7 a shows the maximum separation at interface $\mathrm{C} 1 \mathrm{for}$ different levels of vibration amplitude for the case of $20 \mathrm{Nm}$ bolt torque. Note, as appeared in the time-dependent Figure 6a, the motion is symmetric in the $y$ direction, indicating that an initial gap exists, which with the applied excitation amplitudes never closes. When increasing the input force, the separation increases at the edge away from the bolt; whereas near the bolt, there is no separation for any of the forcing levels. As the maximum and minimum relative displacement are mirrored for all three force levels, it can be assumed that the static gap size at interface $\mathrm{C} 1$ must be a least $9 \mu \mathrm{m}$ at the edge, corresponding to the largest measured displacement occurring at the edge of the interface (cf. Figure 7a).

At interface $\mathrm{C} 3$ the separation is not symmetric in $y$ direction: an opening and closing of the contact interface can be observed (cf. Figure 6b). The measured response at interface C3 is not symmetric (as observed for the response at C1). Instead, what appears to occur is a closing contact with each period of vibration (see Figure 6b). Two maxima per vibration cycle, instead of a maximum and a minimum per vibration cycle (as at C1), supports the occurrence of a closing contact. The two maxima indicate that at the time of zero relative displacements, the upper and lower beam is in contact, as no significant negative relative displacements can be observed at any time at $\mathrm{C} 3$ (in contrast to $\mathrm{C} 1$ ). The closing contact can be interpreted to be the moment when the relative displacement is zero, and the maximum separation in the next half period (with maximum relative displacement at the $-10 \mathrm{~mm}$ position) is may be an effect of the closing contact. The moment of contact is observed to occur over $500 \mu \mathrm{s}$ to $1 \mathrm{~ms}$. As the sampling rate of the data is not sufficiently resolved to inspect the high frequency response of the beam, further investigation is warranted in order to determine the nature of this contact (whether it is an impact or smooth closing). This asymmetric behavior is further highlighted in Figure $7 \mathrm{~b}$, in which the amplitude of the response of interface $\mathrm{C} 3$ is shown. Here, the black curves indicate the maximum separation when the beam is in the lower 


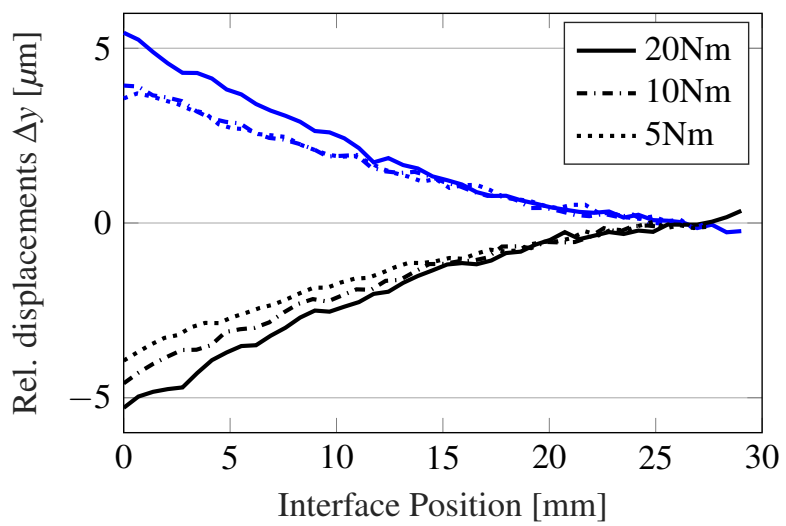

Fig. 8. Maximum relative displacement for different bolt torques, with $2 \mathrm{~N}$ forcing magnitude. Blue lines: Upper bending mode position (cf. Figure 5a). Black lines: Lower bending mode position (half a vibration cycle later).

bending position, and the blue curves indicate the response of the interface half of a period later. The $1 \mathrm{~N}$ excitation is not included as the displacements are below the noise floor and the cyclic behavior cannot be observed. Compared to Figure 7a, the relative displacements are more than five times smaller. Features such as this closing contact are not observable in the quasi-static modeling of this system [5], which partially explains why quasi-static analyses of this and similar systems are unable to account for modal interactions like those observed in dynamic models of the same system (based on the extended periodic motion concept [40]), as well as measurements of similar systems (such as in [38]).

The large difference in the results at $\mathrm{C} 1$ and $\mathrm{C} 3$ suggests that the beams may not be as flat (with features on the order of $10 \mathrm{~s}$ of $\mu \mathrm{ms}$ ) as expected. The local height variations (i.e., the micro-structure of the interface) may have a significant influence on the dynamics. This could possibly explain the difference in results at $\mathrm{C} 1$ and $\mathrm{C} 3$, as the surfaces at $\mathrm{C} 1$ and $\mathrm{C} 3$ can have a significantly different micro-scale topography; this is further investigated in Section 4.4.

\subsection{Influence of bolt torque}

Figure 8 shows the maximum separation at interface $\mathrm{C} 1$ for three different levels of bolt torque $(5,10$ and $20 \mathrm{Nm})$, all with a shaker force of $2 \mathrm{~N}$. Compared to the influence of the vibration a mplitude, the bolt torque does not influence the relative normal displacement along $\mathrm{C} 1$ significantly. Only for the $20 \mathrm{Nm}$ bolt torque measurements the maximum separation is slightly larger than for the two lower torques. This implies that while the bolt torque in this particular case affects the interfacial stiffness [26] (the frequency shifts $2 \mathrm{~Hz}$ from 5 to $20 \mathrm{Nm}$ bolt torque, cf. Figure 5b), it has only a small effect on the local kinematics for this specific interface. The separation observed at the exterior edge (i.e., the portion of the interface furthest from the bolts) is likely due to receding contact [41]: since the contact pressure in the interfaces is concentrated near the bolts, the beams away from the bolts recede from each other due to Poisson strains.

The effect of bolt torque on the local kinematics at $\mathrm{C} 3$ is insignificant (which is why a figure is not in cluded). The relative displacement is ten times smaller than at $\mathrm{C} 1$ and closer to the noise-floor, so if there is a small effect of bolt torque, it cannot be detected with this resolution. A stronger excitation could potentially increase the separation, enabling the effect 


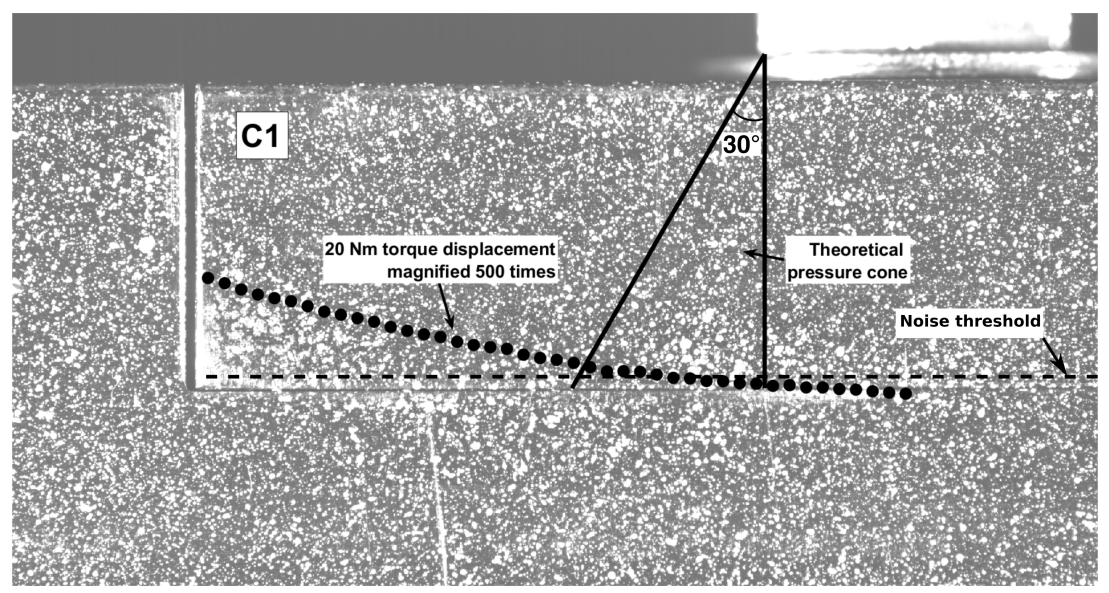

Fig. 9. Illustration of the theoretical pressure cone compared to the separation at interface $\mathrm{C} 1$.

of bolt torque to be determined, but that would risk wear on the surface. At a $2 \mathrm{~N}$ excitation, the separation is effectively the same magnitude as seen in Figure $7 \mathrm{~b}$ for the different bolt torque levels.

\subsection{Pressure cone comparison}

For many applications, a standard theoretical pressure cone is applied to model the expected pressure introduced in a clamped structure by a tightened bolt [42]. A standard angle is $30^{\circ}$ for two perfectly flat surfaces [42]. The separation observed in this work is compared to the pressure cone to see if the standard model comes close to the experimentally observed behavior. Figure 9 is the qualitative comparison between the theoretical pressure cone and the separation measured at $\mathrm{C} 1$ for a $20 \mathrm{Nm}$ bolt torque. The separation is magnified 500 times to visualize how the pressure cone of $30^{\circ}$ overestimates the clamped area for this specific interface. There is separation in the interface within the pressure cone. At C3, the pressure cone underestimates the clamped area, as shown in Figure 7b. Here a separation was only measured at the last $8 \mathrm{~mm}$ of the interface. A possible explanation to the discrepancy again points towards the interface geometry, as the pressure cone assumes perfectly flat interfaces. The comparison is a further indication that two seemingly identical interfaces, $\mathrm{C} 1$ and $\mathrm{C} 3$, can have completely different effectiveness from the bolt clamping and, in turn, the interface behavior.

\subsection{Interface Topography}

To provide further insight into the observed asymmetric behavior of the joint, surface scans are performed on the connecting surfaces of the interfaces at $\mathrm{C} 1$ and $\mathrm{C} 3$, as sketched in Figure 10. A Polytec TMS100 optical surface profilometer with a resolution of $20 \mathrm{~nm}$ is used. The surface scans are visualized in Figure 11. Blue color indicates a rise and red a recess in the surface.

The averages over the width of the surfaces ( $z$ direction) are calculated (with the holes removed from the analysis) to better compare the upper and lower surfaces and are shown in the bottom of Figure 11 for $\mathrm{C} 1$ and $\mathrm{C} 3$. At C1 (Figure 11e), the top beam surface has an average surface recess around $30 \mu \mathrm{m}$ at the right side (where the bolt is mounted). The beams 


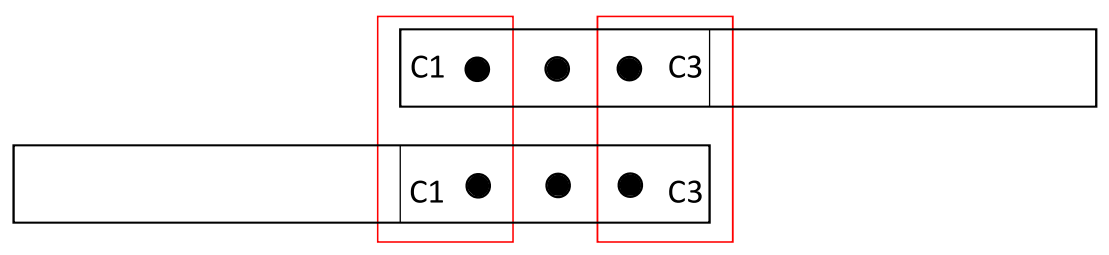

Fig. 10. Scanned surfaces at C1 and C3 after opening of the beam.

have been manufactured to have a mean surface roughness of approximately $1 \mu \mathrm{m}$. The recess indicates that either the manufacturing tolerances have not been met or that the testing has worn the surfaces, which is common in real applications.

The bottom beam is, on average, much smoother and varies by only $3 \mu \mathrm{m}$ over the length. However, the full surface (Figure 11c) should be considered as the lower beam has a recess in the lower left corner, which is smoothed out in the average. If these two surfaces are clamped together, there should be zero or very little gap at the bolt hole, meaning that the blue and black lines in Figure 11e would touch at the right side (i.e., at the bolt hole), which will enforce an initial gap at the exterior edge.

The surface scans at $\mathrm{C} 3$ show no visible rise/recess and are clearly smoother than at $\mathrm{C} 1$. By inspection of the average surface heights, variations of less than $3 \mu \mathrm{m}$ can be seen. The difference in the figures of the average height variation for $\mathrm{C} 1$ and $\mathrm{C} 3$ are pronounced: the top surface at $\mathrm{C} 1$ has a strong rise (roughly $30 \mu \mathrm{m}$ ) preventing the two surfaces from joining close together when the bolt is tightened. In Figure 7a symmetric deflections of $9 \mu \mathrm{m}$ are observed, this is likely due to the initial gap of at least $20 \mu \mathrm{m}$ coming from the uneven interface. In comparison, the surfaces at C3 are smooth, and there is no significant initial gap, which can be an explanation for the asymmetrical gap openings observed in Figure $7 \mathrm{~b}$. The asymmetrical gap opening over a vibration cycle is the surfaces going apart and then closing back together. This can occur because the surfaces are almost smooth. Taken together with the results of Sections 4.1 through 4.5 , it is evident that knowledge of the interface's macro-scale geometry is necessary to have an accurate understanding of the local kinematics and contact patch (and subsequently wear) within an interface. Such understanding of the surface profile and local kinematics is necessary knowledge for modeling the interfacial behavior of the joint correctly; however, the ramifications of the results of Section 4.2 are that this is not enough. The contact and/or gap between the two surfaces is influenced by the surface topography, local kinematics, and mechanics. In Section 4.2, it was shown that higher preloads lead to larger gaps between the surfaces at the edges of the joints. This is explained by a Poisson effect. Consider a thin beam on a rigid (or elastic) half plane. As a point load is applied, Poisson stresses in the beam cause it to peel away from the half plane at its edges. This Poisson contraction is evident here too: larger bolt preloads result in greater Poisson contractions, causing larger gaps between the edges of the interface.

\subsection{Out-of-plane bending mode}

To study the lateral dynamics of the system, the excitation source is rotated such that the beam is excited in the plane orthogonal to the previously measured $y$ displacements. The shaker is remounted to excite the out-of-plane bending mode of 
a) Upper beam $\mathrm{C} 1$

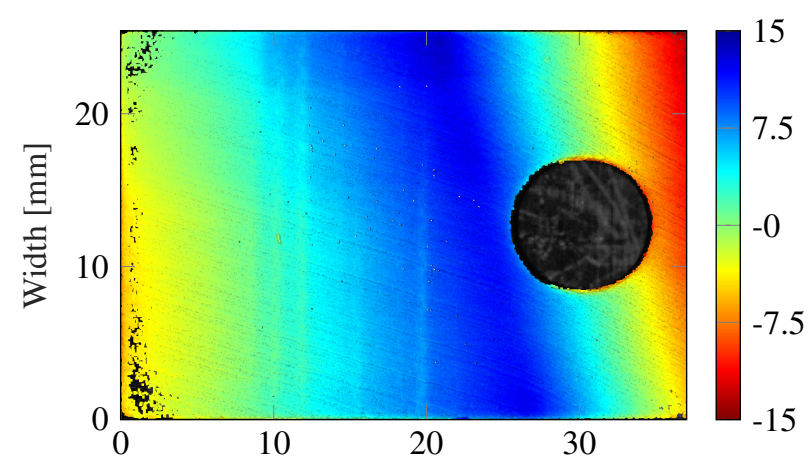

c) Lower beam $\mathrm{C1}$

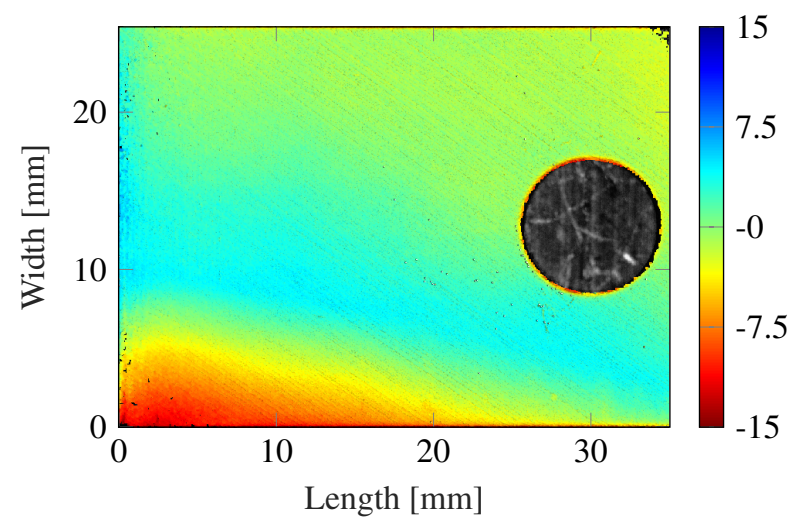

e) Average height variation $\mathrm{C1}$

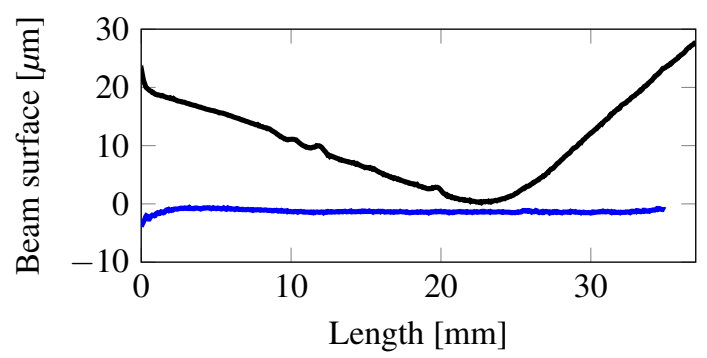

b) Upper beam $\mathrm{C3}$

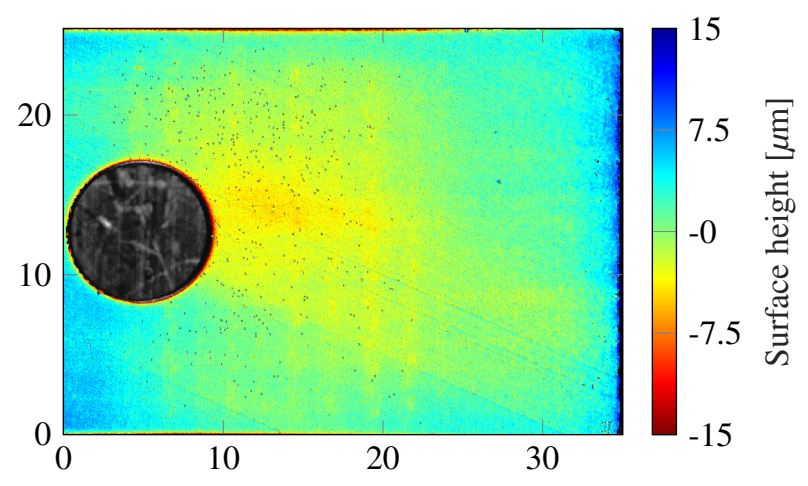

d) Lower beam $\mathrm{C3}$

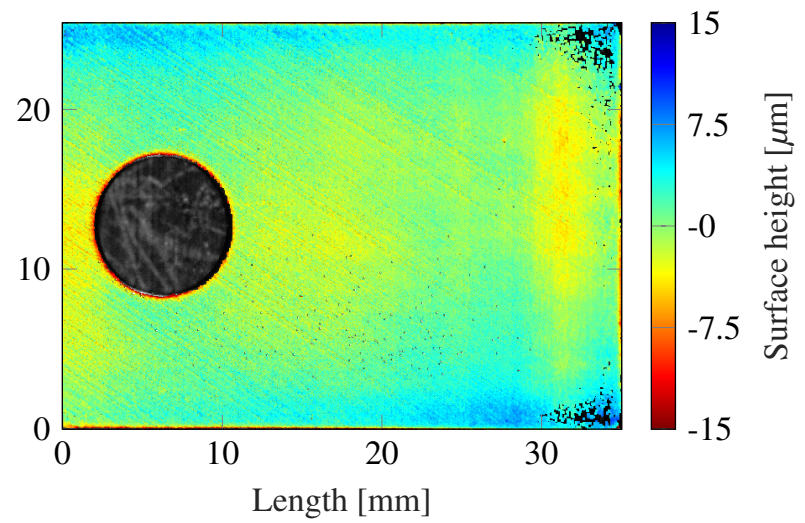

f) Average height variation $\mathrm{C3}$

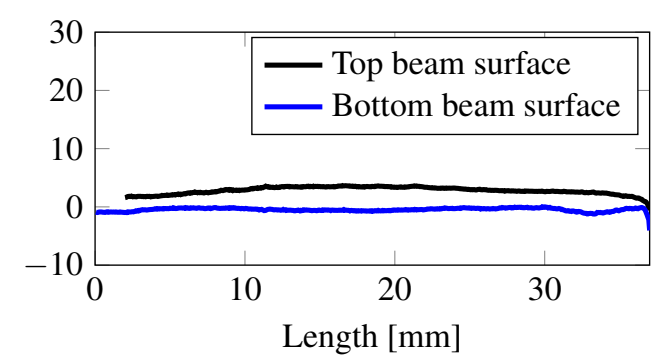

Fig. 11. Raw surface scans of $\mathrm{C} 1$ ((a) and (c)) and C3 ((b) and (d)) for the top ((a) and (b)) and bottom ((c) and (d)) surfaces. Average height variation of the top and bottom beams aligned for (e) $\mathrm{C} 1$ (f) $\mathrm{C} 3$.

$104 \mathrm{~Hz}$ (i.e., in the $z$ direction), which is a slightly stiffer mode than that of the in-plane mode $(80 \mathrm{~Hz})$. In these measurements, the camera is moved to look down on the $x-z$ plane and the DIC technique is used on the images captured in that plane. It must be noted that in this case, the camera does not see the interface edge directly, it will only detect changes in the overlap, $\Delta z$, as the beam vibrates. As for the previous measurements, the global bending motion (in this case out-of-plane bending in the $z$ direction) is canceled out when subtracting the displacements of each beam part, leaving the relative displacement $\Delta z$ as illustrated in Figure 12a. The relative displacement $\Delta z$ of the $x-z$ plane between the two beam parts is therefore constant over the width of the cross-section.

The larger the relative displacement, the more the upper free end of the lap joint moves compared to the lower part when 


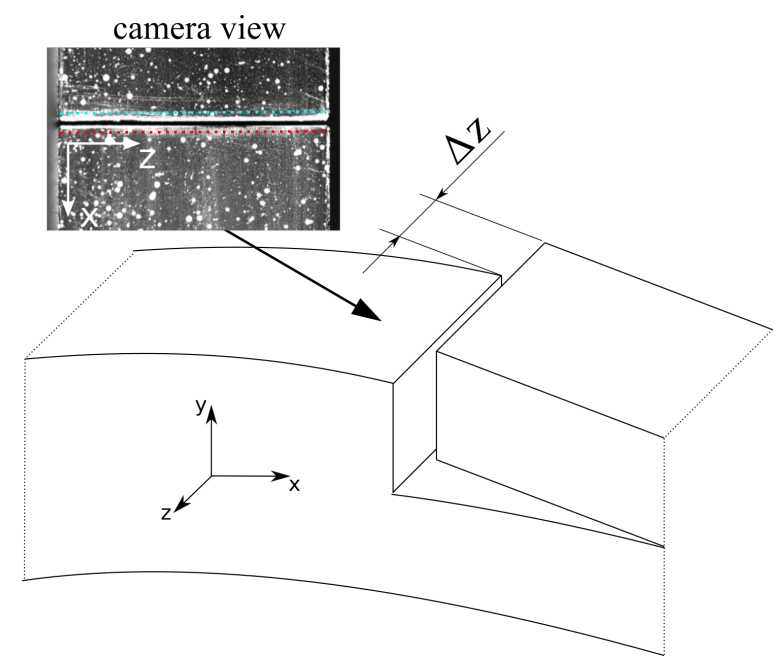

(a)

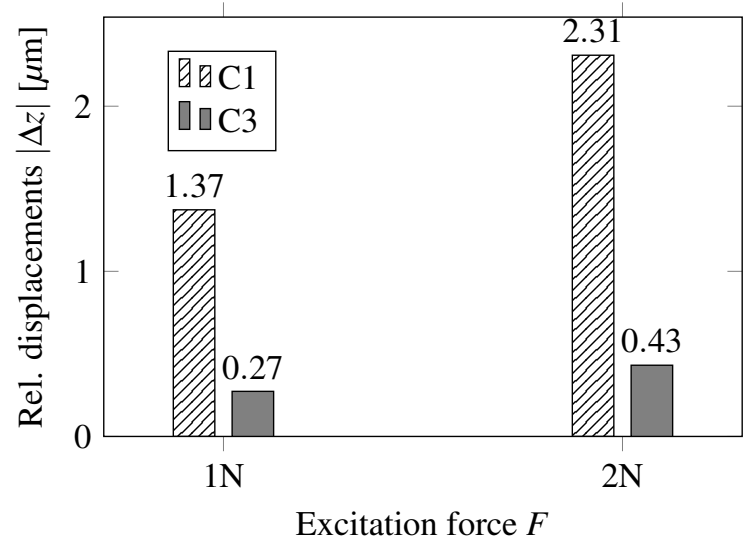

(b)

Fig. 12. Out-of-plane displacement of beam $x, z$-plane. a) Illustration of relative displacement $\Delta z$ with a picture of the camera view from the top of the beam, marked with the DIC subsets (red and blue dots). b) Relative displacement $\Delta z$ for different forcing at $\mathrm{C} 1$ and $\mathrm{C} 3$.

undergoing out-of-plane bending vibrations, as is illustrated in Figure 12a. Figure 12b shows this for both $\mathrm{C} 1$ and $\mathrm{C} 3$ at two different levels of excitation. At $\mathrm{C} 1$, the relative displacement $\Delta z$ is approximately five times larger than at $\mathrm{C} 3$. Increasing the forcing magnifies the relative displacement at $\mathrm{C} 1$ and $\mathrm{C} 3$ with roughly the same percentage.

These measurements support the results of the previous experiments. The bolted lap-joint undergoing bending vibrations, whether it is in the in-plane or out-of-plane direction, exhibit significant local motion, which is also strongly asymmetric. For both directions of bending (i.e., the $y$ and $z$ directions), C1 has the largest magnitude of local motion compared to $\mathrm{C} 3$. This is most likely due to the geometrical difference at the two ends of the bolted joint.

\section{Discussion}

This work had two objectives: first, to investigate if high-speed camera measurements along with DIC could be used to monitor localized motion of an interface, and second, to use the technique to understand the interface mechanisms and how it moves under different operational conditions.

The first objective proved to be possible. By comparing the overall measured bending motion of the upper and lower beams, the local motion at the interface could be extracted. The range of the measured displacement amplitudes of the overall bending motion when undergoing excitation of $1-4 \mathrm{~N}$ is between $0.06-0.25 \mathrm{~mm}$. In all excitation cases, it has been possible to capture relative displacements as small as $0.5 \mu \mathrm{m}$. The speed of the camera and DIC post-processing gave limitations in the resolution of the results. However, the effects of the local motion were still very clear: The two beam parts separate at the free ends and do not behave as a single, homogeneous beam. The exterior edges of the joint move independently, whereas the middle part, between the bolts, is stuck at all times. The exterior edges open and close during a vibration cycle, which can lead to changes in stiffness depending on the excitation amplitude. It was even possible to detect asymmetry 
in the local interface behavior at the left and right end of the beam interface ( $\mathrm{C} 1$ and $\mathrm{C} 3$, respectively). At the left end, the two beams never touch, and there is a cyclic local bending motion of multiple microns amplitude. The local motion at the right end is both qualitatively and quantitatively different. Here, an opening and closing of the contact interface is observed with a closing contact during each period of oscillation. The two beams separate over half of a vibration cycle and then close together. It was possible to measure this cyclic behavior, even though the amplitude was less than one micron. Thus, contrary to assumptions in most joint modeling approaches, the behavior of this geometrically symmetric interface is asymmetric due to micro-scale surface features. This highlights the importance of incorporating the microscale topography of a jointed interface into predictive models.

Further, the static gap confirms previous work on the BRB. In [4] it was found necessary to add a gap in the model to calibrate the numerical predictions to the experimental measurements. Likewise, in [29] measurements with pressure film revealed that the interfaces were not flat (at the micro-scale), and a gap was seen in both e nds. This w ork, together with previous results, suggests that jointed interfaces should be redesigned with robustness to manufacturing tolerances in mind in order to make them more repeatable [13].

The second objective was to understand the interface dynamics and which features could affect it. By increasing the forcing, the amplitude of the local motion increased as well. For both the left and right ends, this is approximately linear: doubling the amplitude of excitation roughly doubled the size of the gap opening, indicating local linear dynamic behavior. Increasing the bolt torque did not significantly change the amplitude of the local gap o penings. As it is hard to control bolt torque (torque wrenches introduces a variability in bolt preload up to 30\% [31]), it is encouraging that bolt torque does not significantly affect the interface kinematics.

In most models, flat surfaces are a ssumed, leading to simple pressure d istributions. A pressure cone gives us a fixed pattern, but this work showed that there was a mismatch between the expected contact area [42] and the experimentally measured contact area. Furthermore, surface scans showed significant variation in micro-scale t opography. In numerical modeling, the implications of this are significant since the real joint has different contact areas, different normal load contributions, and previous results [4] have shown that explicit joint models are particularly sensitive towards these parameters. This is further confirmed by the numerical modeling of non-flat interfaces in [10], in which the numerical predictions showed dramatically different pressure distributions for surfaces with micro-scale topography compared to flat surfaces.

Finally, the analysis of the two separate sides of the interface together with the follow on work performed on a separate beam [27], shows that local motion is extremely dependent on interface geometry. Consequently, the assumption of a perfectly flat and symmetric interface is an oversimplification that would be incompatible with predictive modeling frameworks.. To gain insights into how often and how much micro-scale topography influences dynamics of jointed structures in general, further studies are needed. From these experiments, and those of [27], it is evident that even small variations in micro-scale topography can have a large impact on the local motion. The beam used in these experiments was professionally machined to have a mean roughness of approximately $1 \mu \mathrm{m}$, yet the machining tolerances were significant enough 
to yield qualitatively different behaviors on opposite sides of the interface. For mass production and commercial/industrial applications, it is unrealistic to obtain perfectly flat s urfaces. A dditionally, wear will also change the interface geometry over time [21]. Consequently, to be able to predict dynamic behavior of an interface, either the interface must be measured accurately before testing, or, the design of the interfaces should be changed so that they are more robust (More robust in the sense that small variability in micro-scale surface topography does not completely change the dynamic behavior).

\section{Conclusion}

In this work, the efficacy of digital i mage c orrelation (DIC) has been s tudied for m easuring the local kinematics of a jointed structure. The experimental technique had a resolution of the relative displacements of the interface of $0.5 \mu \mathrm{m}$ during dynamic excitation of the first bending mode of the system (located near $80 \mathrm{~Hz}$ ). This experimental technique has revealed that there are significant local kinematics within the jointed interface that previous modeling efforts had assumed to be inconsequential. The behavior of the interface was found to be strongly dependent upon the local topography of the interface. On one side of the interface of the system studied, due to the curvature unintentionally imparted by the machining process, the two sides of the beam never fully close a $20 \mu \mathrm{m}$ gap during the experiments performed for this work. On the other side, a closing contact appears to occur during each period of oscillation. Additional studies in this work focused on the role of bolt torque and the out-of-plane bending modes. Specific conclusions from this work include:

- The local kinematics of bolted lap joints are significant, and modeling efforts should include these physics in order to model lap joints accurately. This is in direct contradiction to the assumptions behind many common joint modeling techniques.

- The lap joint studied in this work exhibited Poisson effects: as the bolt torque for the joint was increased, the separation between the edges of the interface likewise increased.

- The local topography of the interface (specifically the micro-scale curvature) was strongly influential on determining the dynamic behavior of the lap joint. As the two sides of the interface studied had different micro-scale features, significantly different dynamics were observed for each side of the interface.

One ramification of this work is that the variability observed in most lap joints is likely due to the topography of the interface as this can lead to dynamics that are qualitatively (and quantitatively) different for nominally identical surfaces (i.e. surfaces that are manufactured within machining tolerances). In order to make lap joints more predictable, methods to either reduce the variability in the local topography or to make the system more robust to the effects of the local topography are needed.

\section{Acknowledgements}

The authors would like to thank ROLLS-ROYCE Plc. for their financial support of the NDCSI 2018 summer school, during which this work was conducted. Part of this work was conducted under the EXPERTISE project that received funding 
from the European Union's H2020 research and innovation program under the Marie Skłodowska-Curie grant agreement No 721865. Special thanks goes to Photron for making available a Fastcam SA2 camera for the duration of the project.

\section{References}

[1] Segalman, D., 2005. “A four-parameter iwan model for lap-type joints”. Journal of Applied Mechanics, 72(5), pp. 752760.

[2] Smallwood, D. O., Gregory, D. L., and Coleman, R. G., 2001. "A three parameter constitutive model for a joint which exhibits a power law relationship between energy loss and relative displacement”. In Proceedings of the 72nd Shock and Vibration Symposium, pp. 12-16.

[3] Tol, Ş., and H.N. Özgüven, H., 2015. "Dynamic characterization of bolted joints using frf decoupling and optimization". Mechanical Systems and Signal Processing, 54-55, pp. 124 - 138.

[4] Lacayo, R., Pesaresi, L., Gross, J., Fochler, D., Armand, J., Salles, L., Schwingshackl, C. W., Allen, M., and Brake, M. R. W., 2019. "Nonlinear modeling of structures with bolted joints: A comparison of two approaches based on a time-domain and frequency-domain solver”. Mechanical Systems and Signal Processing, 114, 01.

[5] Balaji, N. N., and Brake, M. R. W., 2019. "The surrogate system hypothesis for joint mechanics". Mechanical Systems and Signal Processing, 126, pp. $42-64$.

[6] Mayes, R. L., 2015. "A craig-bampton experimental dynamic substructure using the transmission simulator method". In Dynamics of Coupled Structures, Volume 4, R. M. M. Allen and D. Rixen, eds., Springer International Publishing, pp. 139-148.

[7] Mignolet, M. P., Song, P., and Wang, X. Q., 2015. “A stochastic iwan-type model for joint behavior variability modeling”. Journal of Sound and Vibration, 349, pp. 289-298.

[8] Petrov, E. P., 2008. "Explicit finite element models of friction dampers in forced response analysis of bladed disks". Journal of Engineering for Gas Turbines and Power, 130(2), p. 022502.

[9] Siewert, C., Panning, L., Wallaschek, J., and Richter, C., 2010. "Multiharmonic forced response analysis of a turbine blading coupled by nonlinear contact forces". Journal of Engineering for Gas Turbines and Power, 132(8), p. 082501.

[10] Balaji, N. N., Chen, W., and Brake, M. R., 2020. “Traction-based multi-scale nonlinear dynamic modeling of bolted joints: Formulation, application, and trends in micro-scale interface evolution". Mechanical Systems and Signal Processing, 139, p. 106615.

[11] Iranzad, M., and Ahmadian, H., 2012. "Identification of nonlinear bolted lap joint models". Computers and Structures, 96-97, pp. 1-8.

[12] Gaul, L., and Lenz, J., 1997. "Nonlinear dynamics of structures assembled by bolted joints". Acta Mechanica, 125(1), pp. 169-181.

[13] Brake, M. R. W., 2017. The Mechanics of Jointed Structures: Recent Research and Open Challenges for Developing 
Predictive Models for Structural Dynamics. Cham: Springer International Publishing.

[14] Eriten, M., Kurt, M., Luo, G., Michael McFarland, D., Bergman, L. A., and Vakakis, A. F., 2013. “Nonlinear system identification of frictional effects in a beam with a bolted joint connection”. Mechanical Systems and Signal Processing, 39(1-2), pp. 245-264.

[15] Chen, W., and Deng, X., 2005. "Structural damping caused by micro-slip along frictional interfaces". International Journal of Mechanical Sciences, 47(8), pp. 1191-1211.

[16] Ouyang, H., Oldfield, M. J., and Mottershead, J. E., 2006. "Experimental and theoretical studies of a bolted joint excited by a torsional dynamic load”. International Journal of Mechanical Sciences, 48(12), pp. 1447-1455.

[17] Čelič, D., and Boltežar, M., 2008. "Identification of the dynamic properties of joints using frequency-response functions". Journal of Sound and Vibration, 317(1-2), pp. 158-174.

[18] Krack, M., Bergman, L. A., and Vakakis, A. F., 2016. "On the efficacy of friction damping in the presence of nonlinear modal interactions". Journal of Sound and Vibration, 370, pp. 209-220.

[19] Ma, X., Bergman, L., and Vakakis, A., 2001. "Identification of bolted joints through laser vibrometry". Journal of Sound and Vibration, 246(3), pp. 93573, 441-460.

[20] Ruffini, V., Schwingshackl, C. W., and Green, J. S., 2013. "LDV measurement of local nonlinear contact conditions of flange joint”. Conference Proceedings of the Society for Experimental Mechanics Series, 1, pp. 159-168.

[21] Fantetti, A., Tamatam, L. R., Volvert, M., Lawal, I., Liu, L., Salles, L., Brake, M. R. W., Schwingshackl, C. W., and Nowell, D., 2019. “The impact of fretting wear on structural dynamics: Experiment and simulation”. Tribology International, 138, pp. 111-124.

[22] Pesaresi, L., Stender, M., Ruffini, V., and Schwingshackl, C. W., 2017. "DIC measurement of the kinematics of a friction damper for turbine applications”. In Dynamics of Coupled Structures, Volume 4. Springer, pp. 93-101.

[23] Lall, P., Panchagade, D. R., Iyengar, D., Shantaram, S., and Schrier, H., 2009. "High speed digital image correlation for transient-shock reliability of electronics". Ieee Transactions on Components and Packaging Technologies, 32(2), pp. 378-395.

[24] Comer, A. J., Katnam, K. B., Stanley, W. F., and Young, T. M., 2013. "Characterising the behaviour of composite single lap bonded joints using digital image correlation”. International Journal of Adhesion and Adhesives, 40, pp. 215 - 223.

[25] Kumar, R. L. V., Bhat, M. R., and Murthy, C. R. L., 2013. "Experimental analysis of composite single-lap joints using digital image correlation and comparison with theoretical models". Journal of Reinforced Plastics and Composites, 32(23), pp. 1858-1876.

[26] Brake, M. R. W., Schwingshackl, C. W., and Reuß, P., 2019. “Observations of variability and repeatability in jointed structures". Mechanical Systems and Signal Processing, 129, pp. 282 - 307.

[27] Chen, W., Jin, M., Lawal, I., Brake, M. R. W., and Song, H., 2019. "Measurement of slip and separation in jointed structures with non-flat interfaces”. Mechanical Systems and Signal Processing, 134, p. 106325. 
[28] Lawal, I., Shah, S., Gonzalez-Madrid, M., Hu, T., Schwingshackl, C. W., and Brake, M. R. W., 2019. “The effect of non-flat interfaces on system dynamics". Nonlinear Dynamics, Volume 1, pp. 187-197.

[29] Dossogne, T., Jerome, T. W., Lancereau, D. P. T., Smith, S. A., Brake, M. R. W., Pacini, B. R., Reuß, P., and Schwingshackl, C. W., 2017. "Experimental assessment of the influence of interface geometries on structural dynamic response". In Dynamics of Coupled Structures, Volume 4, R. M. M.S. Allen and D. Rixen, eds. Springer International Publishing, Cham, pp. 255-261.

[30] Seeger, B., Butaud, P., Baloglu, M. V., Du, F., Brake, M. R., and Schwingshackl, C. W., 2019. "In situ measurements of interfacial contact pressure during impact hammer tests". Conference Proceedings of the Society for Experimental Mechanics Series, 1, pp. 225-236.

[31] Bickford, J. H., 2008. Introduction to the design and behavior of bolted joints / John H. Bickford ; Vol. 1: Non-gasketed joints. Boca Raton: CRC Press.

[32] Reu, P., 2015. "Introduction to digital image correlation: Best practices and applications". Experimental Techniques, 36(1), pp. 3-4.

[33] Turner, D., 2015. "Digital image correlation engine (DICe) reference manual”. Sandia Report, SAND2015-10606 O.

[34] Reu, P., 2012. "Stereo-rig design: Creating the stereo-rig layout-part 1". Experimental Techniques, 36(5), pp. 3-4.

[35] Neggers, J., Blaysat, B., Hoefnagels, J. P. M., and Geers, M. G. D., 2016. “On image gradients in digital image correlation”. International Journal for Numerical Methods in Engineering, 105(4), 1, pp. 243-260.

[36] Turner, D. Z., 2016. "An overview of the gradient-based local DIC formulation for motion estimation in DICe”. Sandia Report, SAND2016-7360 R.

[37] Keys, R., 1981. "Cubic convolution interpolation for digital image processing”. IEEE Transactions on Acoustics, Speech, and Signal Processing, 29(6), Dec, pp. 1153-1160.

[38] G. Kosova, M. Jin, M. Cenedese, W. Chen, A. Singh, D. Jana, M. R. W. Brake, C. W. Schwingshackl, K. J. Moore, and Noël, J.-P. "Nonlinear System Identification of a Jointed Structure Using Full-Field Data: Part II Analysis”. In IMAC XXXVIII A Conference and Exposition on Structural Dynamics.

[39] A. Singh, W. Chen, D. Jana, M. Jin, M. Cenedese, M. R. W. Brake, C. W. Schwingshackl, K. J. Moore, and J.-P. Noël, and Noël, J.-P. “Nonlinear System Identification of a Jointed Structure Using Full-Field Data: Part 1 Experimental Investigation”. In IMAC XXXVIII A Conference and Exposition on Structural Dynamics.

[40] Krack, M., 2015. "Nonlinear modal analysis of nonconservative systems: Extension of the periodic motion concept". Computers and Structures, 154, pp. 59-71.

[41] Flicek, R. C., 2015. "Analysis of complete contacts subject to fatigue”. PhD thesis, Oxford University, UK.

[42] Budynas, R. G., and Nisbett, J. K., 2015. Shigley's mechanical engineering design. McGraw-Hill Education. 


\section{List of Figures}

1 A sketch of the extended Brake-Reuß beam used for the measurements. The bolt tightening order is shown. A gap of $0.2 \mathrm{~mm}$ is ensured on the left side of the interface by means of the shim. . . . . . . . . . 5

2 (a) Real experimental setup. (b) scheme of the two configurations, in plane bending ( $y$ direction) and out-ofplane bending ( $z$ direction), with the beam and shaker suspended with fishing line . . . . . . . . . 6

3 A sample frame from the high-speed camera. The three different areas of interest $(\mathrm{C} 1, \mathrm{C} 2, \mathrm{C} 3)$ are shown. The relative displacements of the interfaces (defined by the subsets in the red and blue line along the contactline) will be measured. A zoom-in of a typical $61 \times 61$ pixel subset and the principle of subsets, step size

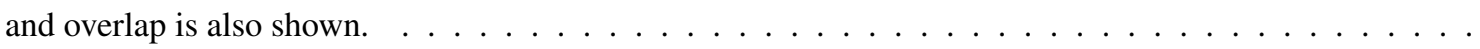

4 (a) The standard deviation of the relative displacement measurement for different subset sizes. (b) Illustration

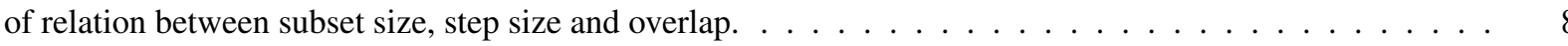

5 a) Illustration of the first bending mode of the beam. b) Frequency response functions measured by low level hammer impact around the first bending natural frequency at different bolt torque levels . . . . . . . . 10 Relative $y$ displacement field of the horizontal interfaces. at a) $\mathrm{C} 1$, b) $\mathrm{C} 3$, and c) $\mathrm{C} 2 \ldots \ldots \ldots$

7 Maximum relative displacement for different forcing magnitudes, with $20 \mathrm{Nm}$ bolt torque. Blue lines: Upper bending mode position (cf. Figure 5a). Black lines: Lower bending mode position (half a vibration cycle later). a) Interface $\mathrm{C} 1$. b) Interface $\mathrm{C} 3 \ldots \ldots \ldots \ldots \ldots \ldots \ldots$

8 Maximum relative displacement for different bolt torques, with $2 \mathrm{~N}$ forcing magnitude. Blue lines: Upper bending mode position (cf. Figure 5a). Black lines: Lower bending mode position (half a vibration cycle later). 13

$9 \quad$ Illustration of the theoretical pressure cone compared to the separation at interface C1 . . . . . . . . . 14

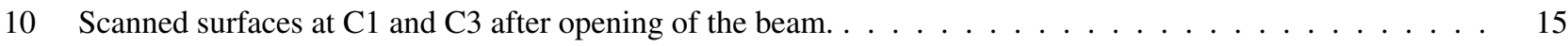

11 Raw surface scans of C1 ((a) and (c)) and C3 ((b) and (d)) for the top ((a) and (b)) and bottom ((c) and (d)) surfaces. Average height variation of the top and bottom beams aligned for (e) $\mathrm{C} 1$ (f) C3. . . . . . . . 16

12 Out-of-plane displacement of beam $x, z$-plane. a) Illustration of relative displacement $\Delta z$ with a picture of the camera view from the top of the beam, marked with the DIC subsets (red and blue dots). b) Relative displacement $\Delta z$ for different forcing at $\mathrm{C} 1$ and $\mathrm{C} 3 \ldots \ldots \ldots \ldots \ldots \ldots \ldots \ldots$ 


\section{List of Tables}

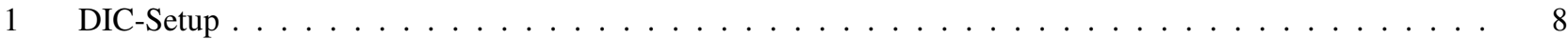

